
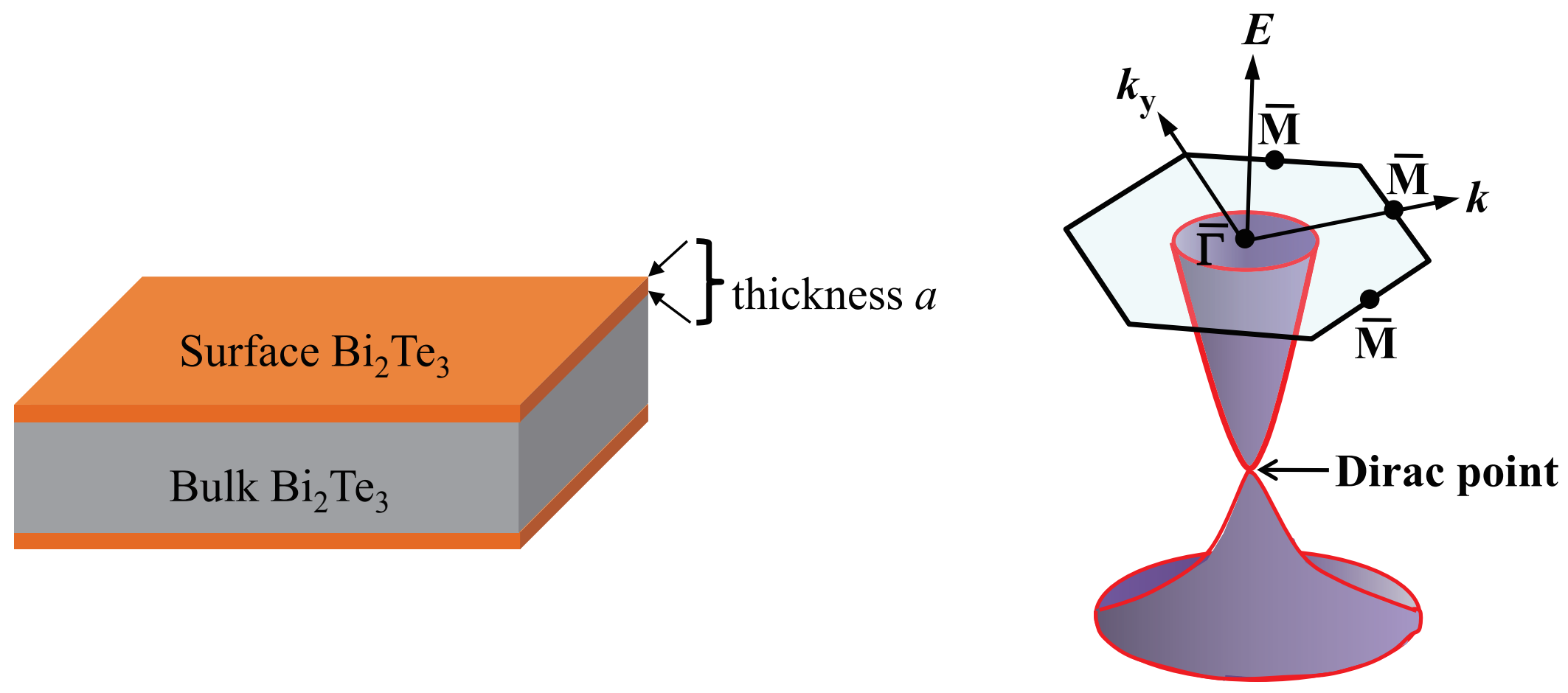

Fig. 1: Schematic picture of the surface layer with length scale $a$ and Dirac cone of the surface layer 

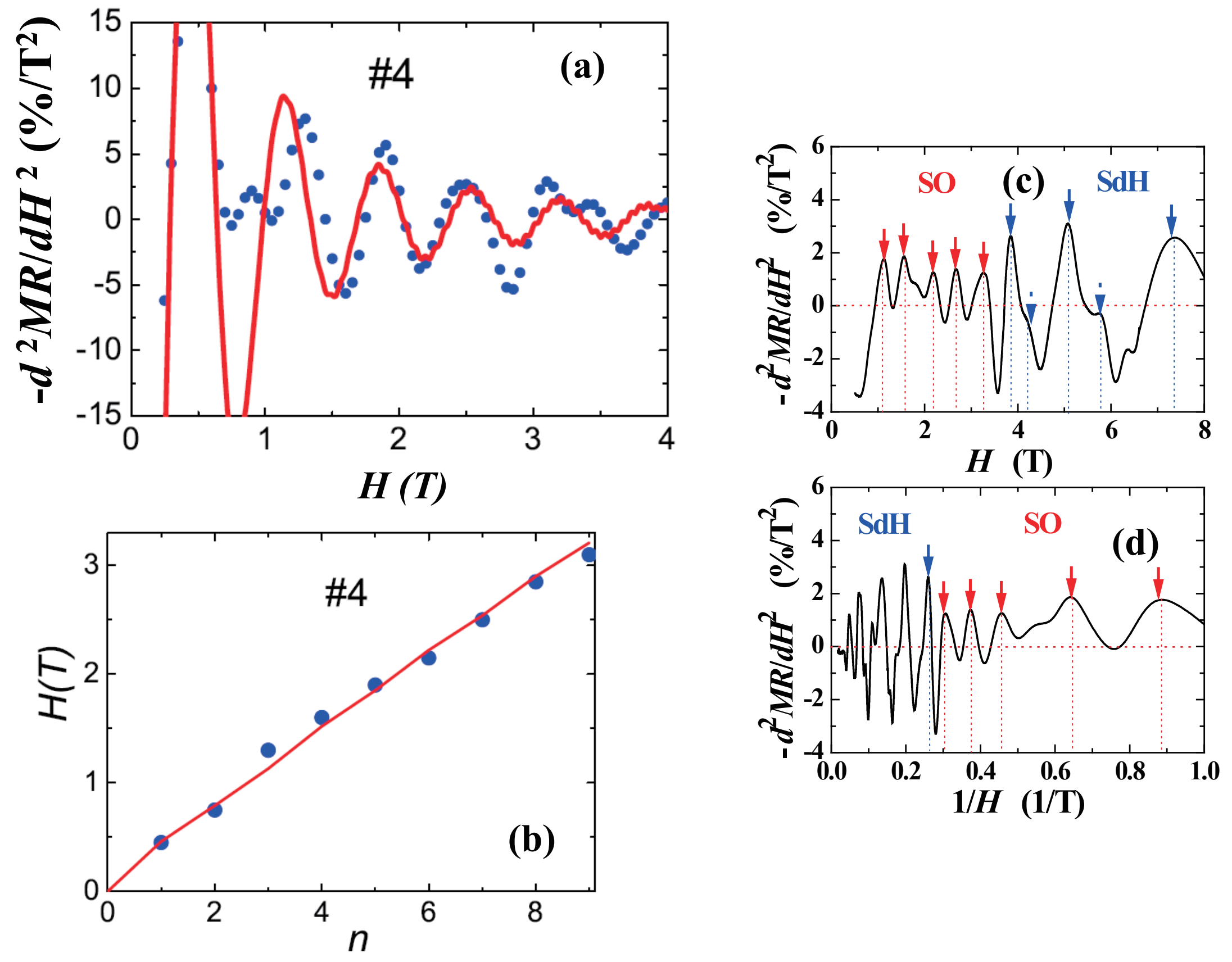

Fig. 2: Sonderheimer oscillation (SO) in magnetoresistance 

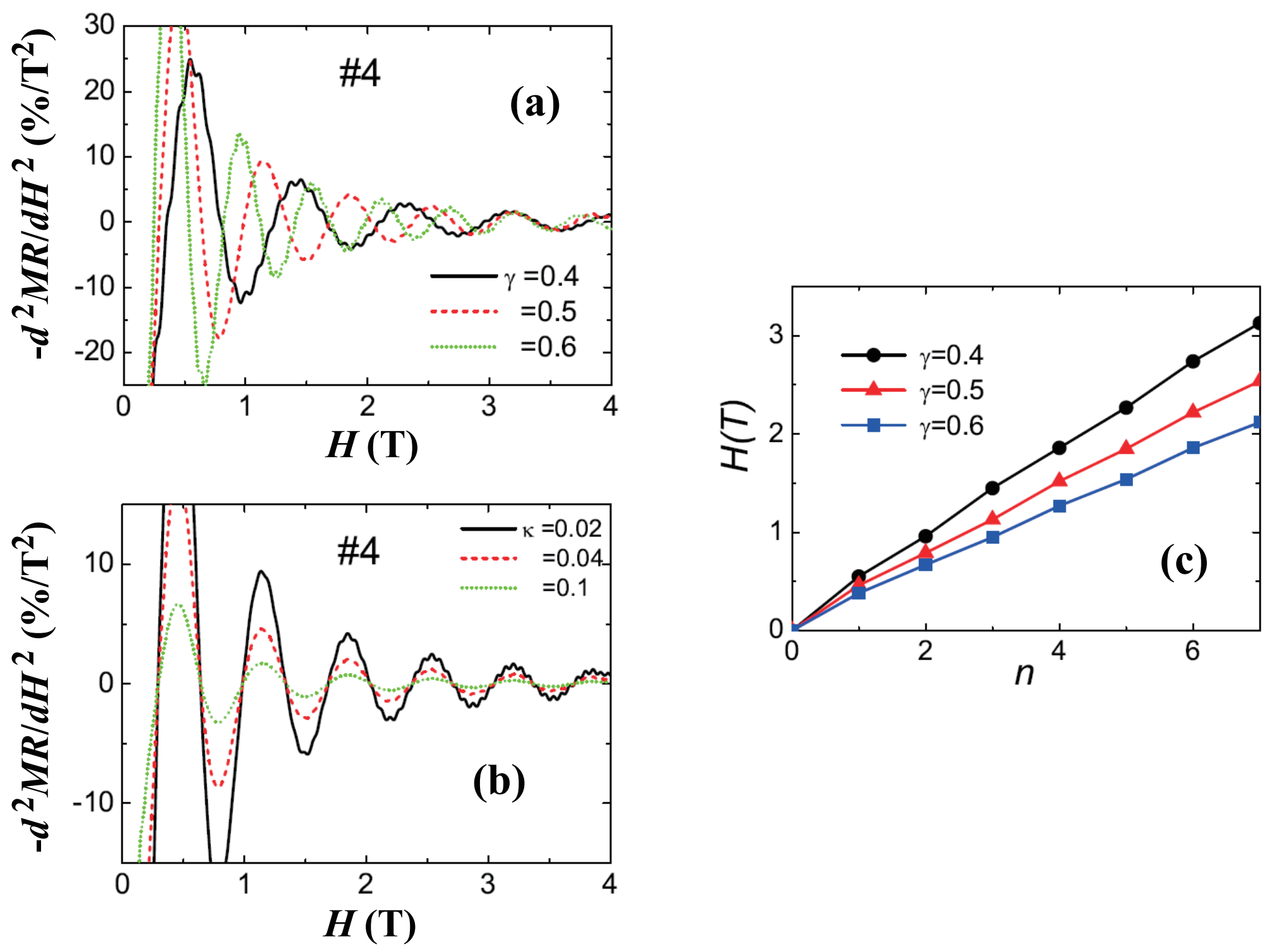

Fig. 3: Dependence of SO on the Fermi energy and the disorder strength 

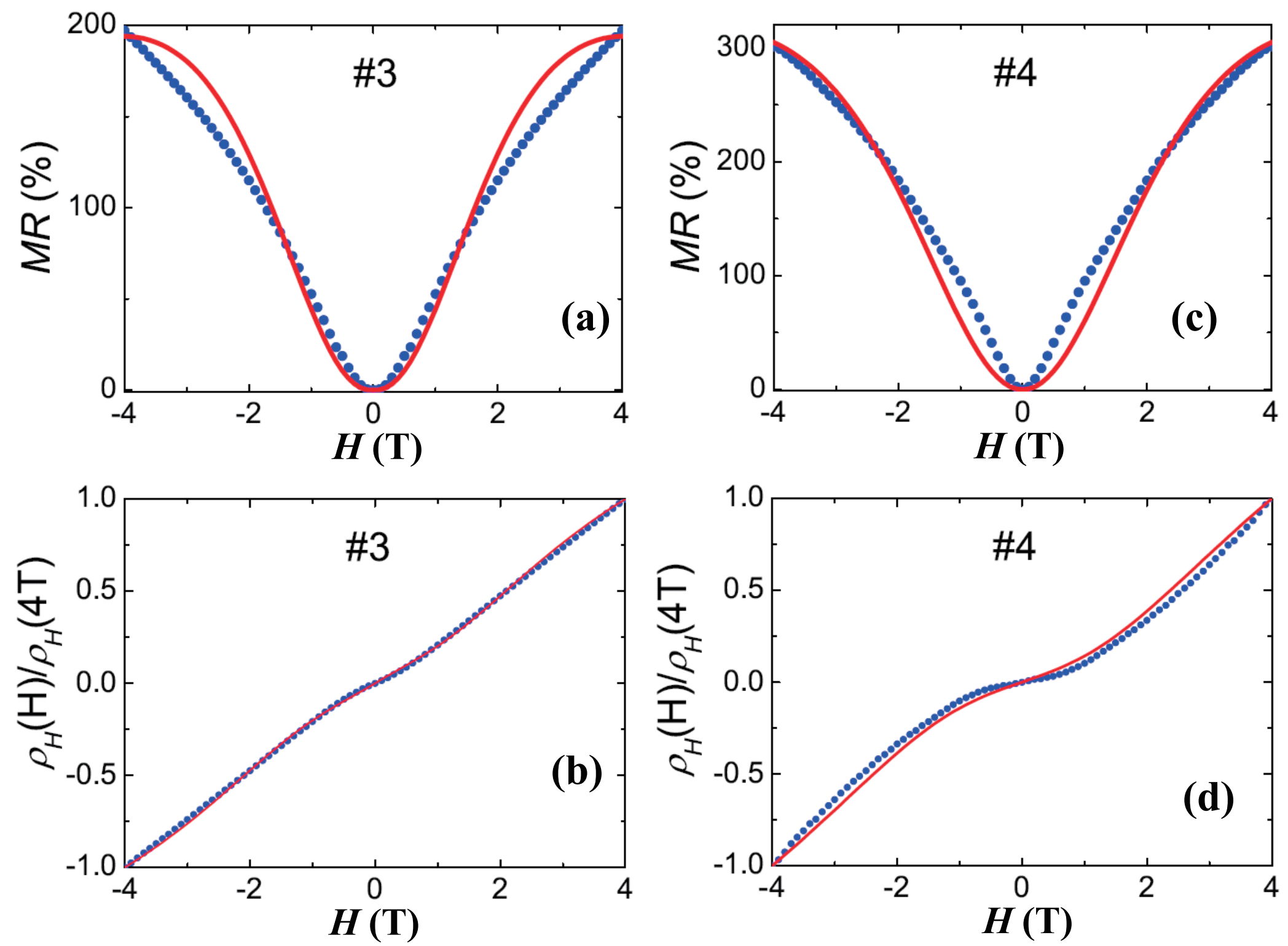

Fig. 4: MR and Hall resistance of sample \#3 and \#4 with theoretical fitting curves 


\title{
Sondheimer Oscillation as a Fingerprint of Surface Dirac Fermions
}

\author{
Heon-Jung Kim ${ }^{1, *}$, Ki-Seok Kim ${ }^{2,3}$, Mun Dae Kim ${ }^{4}$, S.-J. Lee ${ }^{5}$, J.-W. Han ${ }^{1}$, \\ A. Ohnishi ${ }^{5}$, M. Kitaura ${ }^{5}$, and M. Sasaki ${ }^{5, \dagger}$, A. Kondo ${ }^{6}$, and K. Kindo ${ }^{6}$ \\ ${ }^{1}$ Department of Physics, College of Natural Science, \\ Daegu University, Gyeongbuk 712-714 Republic of Korea \\ ${ }^{2}$ Asia Pacific Center for Theoretical Physics, \\ POSTECH, Pohang, Gyeongbuk 790-784, Korea \\ ${ }^{3}$ Department of Physics, POSTECH, \\ Pohang, Gyeongbuk 790-784, Korea \\ ${ }^{4}$ Institute of Physics and Applied Physics, \\ Yonsei University, Seoul 120-749, Korea \\ ${ }^{5}$ Department of Physics, Faculty of Science, Yamagata University, \\ Kojirakawa 1-4-12 Yamagata, 990-8560, Japan \\ ${ }^{6}$ Institute for Solid State Physics, University of Tokyo, \\ Kashiwanoha 5-1-5, Kashiwa, Chiba 277-8581 Japan
}

(Dated: October 27, 2018) 


\begin{abstract}
Topological states of matter challenge the paradigm of symmetry breaking, characterized by gapless boundary modes and protected by the topological property of the ground state. Recently, angle-resolved photoemission spectroscopy (ARPES) has revealed that semiconductors of $\mathrm{Bi}_{2} \mathrm{Se}_{3}$ and $\mathrm{Bi}_{2} \mathrm{Te}_{3}$ belong to such a class of materials. Here, we present undisputable evidence for the existence of gapless surface Dirac fermions from transport in $\mathrm{Bi}_{2} \mathrm{Te}_{3}$. We observe Sondheimer oscillation in magnetoresistance (MR). This oscillation originates from the quantization of motion due to the confinement of electrons within the surface layer. Based on Sondheimer's transport theory, we determine the thickness of the surface state from the oscillation data. In addition, we uncover the topological nature of the surface state, fitting consistently both the non-oscillatory part of MR and the Hall resistance. The side-jump contribution turns out to dominate around 1 $\mathrm{T}$ in Hall resistance while the Berry-curvature effect dominates in $3 \mathrm{~T} \sim 4 \mathrm{~T}$.
\end{abstract}

PACS numbers: 
Symmetry breaking is the paradigm in not only classifying quantum states of matter but also describing phase transitions between them, where the correlation length of fluctuations between local order parameters diverges at the critical point of a continuous transition [1, 2]. On the other hand, topological states of matter are classified by topological quantum numbers [3, 4], associated with gapless boundary electronic states and are protected from the topological properties of the ground state [5, 6]. Instead of a divergence in correlation length, topological phase transitions are accompanied by changes of the gapless boundary modes. One possible mechanism for this phenomenon is the length scale of the boundary state becomes of the same order as the bulk size, causing the gapless modes in opposite boundaries to be mixed and making such boundary modes gapped [7]. In this case, the length scale for the boundary mode plays basically the same role as the correlation length for the phase transition, which is the fundamental length scale for a topological phase (Fig. 1a).

Recently, the semiconductor material $\mathrm{Bi}_{2} \mathrm{Se}_{3}$ and $\mathrm{Bi}_{2} \mathrm{Te}_{3}$ have been verified to be three dimensional topological insulators. These insulators are regarded as a novel quantum state of matter [8 11], where gapless surface electrons are uncovered in ARPES [12 14]. Although the surface states in these materials and the electronic structure in graphene are described by Dirac theory (Fig. 1b), the surface state of topological insulators is profoundly different from the electronic structure of graphene [15]. This difference originates from the absence of both the sublattice symmetry and valley degeneracy. The direction of spin is locked with that of the momentum in surface Dirac electrons [16]. This completely suppresses backscattering due to time-reversal invariant impurities, allowing a super-metallic state [17].

In this letter we focus on MR and Hall measurements, both of which are of high importance for the fundamental understanding and practical applications of topological insulators. We observe an oscillatory behavior in MR at low magnetic fields of up to $4 \mathrm{~T}$. This behaviour can be identified as Sondheimer oscillation [18], where the oscillation period is linearly proportional to the magnetic field (Fig. 2). Sondheimer's transport theory [18], combined with Dirac dispersion, enables us to determine the fundamental length scale from our experimental data, which turns out to be about 5 atomic layers. In this respect the Sondheimer oscillation can be regarded as an inevitable result of the surface state. The nature of the surface state can be explained by the single Dirac-fermion theory. This explains not only the non-oscillatory part of MR but also the topological properties implicit in Hall resistance 
in a quantitative and consistent way (Fig. 4). In particular, we show that the Hall resistance of the surface state is dominated by the side-jump around $1 \mathrm{~T}$ and below while the Berry-curvature contribution is dominant at higher fields (Fig. 5 in SI III).

In our measurements we used defect-controlled $\mathrm{Bi}_{2} \mathrm{Te}_{3}$ single crystals. Usually, as-grown $\mathrm{Bi}_{2} \mathrm{Te}_{3}$ single crystals are p-doped because of the anti-site defects in Bi sites [27]. In order to tune the Fermi level, we have controlled the amount of defects by adding extra Bi or Te; the doped $\mathrm{Bi}$ tends to increase the anti-site defects, while the doped Te tends to decrease them. Based on this strategy, we have succeeded in growing a range of $\mathrm{Bi}_{2} \mathrm{Te}_{3}$ single crystals, from fully p-doped to fully n-doped regions. Though rare, we have obtained almost insulating $\mathrm{Bi}_{2} \mathrm{Te}_{3}$ single crystals. The chance for obtaining these samples is around $3 \%$. The carrier type is determined by thermoelectric power at room temperature and also by Hall sign measured at $4.2 \mathrm{~K}$. Samples $\sharp 1$ and $\sharp 2$ are hole-doped while sample $\sharp 5$ is electron-doped. Samples $\sharp 3$ and $\sharp 4$ are close to insulators, as such, they are expected to show the topological properties of the surface state well. See the supplementary material (SI I) for our sample preparation.

Magnetoresistance (MR) and Hall effect measurements have been carried out by a sixprobe method at $4.2 \mathrm{~K}$ using a superconducting magnet up to $4 \mathrm{~T}$ and a $60 \mathrm{~T}$ pulse magnet at ISSP in Tokyo university up to $55 \mathrm{~T}$. Here, the direction of the magnetic fields is applied perpendicular to the naturally cleaved plane, on which the current is applied. For the MR and Hall measurements, we carefully contacted the lead wires reduce the induction noise for high-field pulse-magnet experiments. We have taken the anti-symmetrized and the symmetrized parts as Hall and longitudinal resistances, respectively.

ARPES has unveiled only a single Dirac-fermion band at the surface of $\mathrm{Bi}_{2} \mathrm{Te}_{3}[13,14]$. Therefore, in order to analyze our experimental data, we introduce an electromagnetic vector potential $\overrightarrow{\boldsymbol{A}}$ and a Zeeman term into the single Dirac-fermion theory. In addition, we take into account impurity scattering at the level of a Born approximation. Our theoretical analysis reveals that the orbital contribution or the effect of the vector potential on experimental data is irrelevant in the region of magnetic fields below $4 \mathrm{~T}$ (SI IV). However, its influence on MR and Hall resistance can appear at higher magnetic fields, where Landau levels are fully developed.

We model the surface state as a thin layer with thickness $a$ (Fig. 1a), which is used in the Boltzmann equation (SI II). This approach is essentially the same as what Sondheimer used 
with metallic thin films except for the band structure, where non-relativistic electrons are replaced with Dirac fermions in the presence of the Zeeman term. The main consequence of this is the quantization of motion along the direction normal to the surface which produces an oscillatory component of MR. In our measurements at magnetic fields below $4 \mathrm{~T}$, the periodicity observed is linearly proportional to $H$ [18]. This oscillation is distinguished from Shubnikov-de Haas oscillation due to the formation of Landau levels, where the periodicity is proportional to $1 / H$. See Fig. 2 .

Sondheimer's transport theory [18] with the Dirac dispersion gives the following expression for the oscillating part of the longitudinal resistivity

$$
\frac{\rho(H, T)}{\rho(0,0)}=\frac{1}{\kappa} \Re \phi(s),
$$

where the kernel

$$
\frac{1}{\phi(s)}=\frac{1}{s}-\frac{3}{8 s^{2}}+\frac{3}{2 s^{2}} \int_{1}^{\infty} d u e^{-s u}\left(\frac{1}{u^{3}}-\frac{1}{u^{5}}\right)
$$

with $s=\kappa+i \beta$ results from the distribution in the hard-wall boundary condition for the $z$-direction (SI II). Two parameters appear in this expression, $\kappa=a / l$ and $\beta=a / r_{c}$, where $l$ is the mean free path and $r_{c}$ is the magnetic length (proportional to $\left.1 / H\right)$ ). $a$ is the thickness of the surface state. This transport theory produces the $H$-linear periodicity. It is worth noting that the periodicity in MR depends only on $\beta$ while $\kappa$ modifies the amplitude of oscillation as shown in Fig. 3.

Our experimental data shows that the Sondheimer oscillation turns into the Shubnikov-de Haas oscillation above $3 \mathrm{~T} \sim 4 \mathrm{~T}$ (Fig. 2). This is consistent with several recent transport measurements that show Shubnikov-de Hass oscillation beginning at around $4 \mathrm{~T}$ [19 22]. The appearance of the Shubnikov-de Haas oscillation is the origin for the mismatch of the oscillation amplitude.

We point out that $\beta$ can be written as $\beta=\frac{1}{2}\left(k_{f} a\right) h\left(k_{f} / \bar{k}\right)$ with the surface thickness $a$, where $h=\hbar \omega_{L} / E_{F}$ is the ratio of the Zeeman energy $\hbar \omega_{L}$ to the Fermi energy $E_{F}$ at the surface, and $k_{f}$ and $\bar{k}$ are the Fermi wave-vector and the average momentum, respectively. See the supplementary information for details. An important aspect is that $h$ also enters both the non-oscillatory part of MR and Hall resistance. Therefore, the actual value of $h$ influences not only the periodicity of the Sondheimer oscillation but also both the nonoscillatory part of MR and Hall resistance. Precisely speaking, the surface thickness cannot 
be determined from the Sondheimer oscillation alone. Combined with the longitudinal and transverse resistances, we can optimize the thickness $a$ and the coefficient $\gamma$ simultaneously (SI II), where $\gamma$ is the ratio $\hbar \omega_{L} / E_{F}$ at $H=1 \mathrm{~T}$. It is interesting to note that the optimized $\gamma$ almost coincides with the bulk value. Furthermore, this $\gamma$ value seems to be common to both $\mathrm{Bi}_{2} \mathrm{Te}_{3}$ and $\mathrm{Bi}_{2} \mathrm{Se}_{3}$ at a given field (SI II). Our fitting for the oscillatory part of MR, performed consistently for both the non-oscillating part of MR and Hall resistance, gives a result for the surface thickness of approximately 5 atomic layers. This is quite remarkable in that this value is consistent with that in MBE-grown $\mathrm{Bi}_{2} \mathrm{Te}_{3}$ thin films [23].

Next, we focus on the topological nature of the surface state. Strictly, the role of the single Dirac-fermion theory is not essential in Sondheimer oscillation although Dirac dispersion is utilized. It might be the case that the surface state is realized due to the good surface quality of our samples. However, we will show that the Sondheimer oscillation is a signature of surface Dirac electrons in $\mathrm{Bi}_{2} \mathrm{Te}_{3}$, verifying that the Hall resistance originates from the anomalous Hall effect of Dirac theory. In addition, we show that the side-jump contribution dominates at low magnetic fields and the Berry-curvature effect dominates at high magnetic fields.

The single Dirac-fermion theory gives an analytic expression for the longitudinal conductance [24]

$$
\sigma_{x x}(H, T \rightarrow 0)=\alpha \frac{e^{2}}{2 \pi \hbar} \frac{\sqrt{1+h^{2}}}{1+4 h^{2}}
$$

where $\alpha \equiv \frac{4 n e^{2}(2 \pi \hbar)^{3} v_{f}}{m^{*} n_{I}\left[V_{I}^{(0)}\right]^{2} k_{f}}$ is a dimensionless parameter which measures the strength of disorder with an impurity density $n_{I}$ and an impurity potential $V_{I}^{(0)}$, while $v_{f}$ is the Dirac-fermion velocity with the Fermi momentum $k_{f} . h$ is the dimensionless magnetic field, introduced in Sondheimer oscillation.

The same Dirac theory results in Hall conductance [24]

$$
\begin{aligned}
\sigma_{x y}(H, T \rightarrow 0) & =\sigma_{x y}^{F S}(H, T \rightarrow 0)+\sigma_{x y}^{A}(H, T \rightarrow 0), \\
\sigma_{x y}^{A}(H, T \rightarrow 0) & =\sigma_{x y}^{B}(H, T \rightarrow 0)+\sigma_{x y}^{S J}(H, T \rightarrow 0) \\
& +\sigma_{x y}^{S K}(H, T \rightarrow 0) .
\end{aligned}
$$

Hall conductance consists of two contributions. The first results from electrons near the Fermi surface, referred as normal Hall conductance while the second contribution comes from a fully occupied band, called anomalous Hall conductance. We use the normal Hall 
conductance from the Boltzman equation approach for the Sondheimer oscillation, which is given by

$$
\sigma_{x y}^{F S}(H, T \rightarrow 0)=\kappa \sigma_{x x}(H, T \rightarrow 0) \frac{\Im \phi(s)}{[\Re \phi(s)]^{2}+[\Im \phi(s)]^{2}}
$$

The anomalous Hall conductance is also composed of two contributions. The first comes purely from the topological character of the band structure, identified with the Berrycurvature term $\sigma_{x y}^{B}(H, T \rightarrow 0)=-\frac{e^{2}}{4 \pi \hbar} \frac{h}{\sqrt{1+h^{2}}}$, while the second originates from scattering with disorder in the presence of the spin-orbit interaction. This disorder contribution is separated into the side-jump term $\sigma_{x y}^{S J}(H, T \rightarrow 0)=-\frac{e^{2}}{4 \pi \hbar} \frac{h}{\sqrt{1+h^{2}}}\left\{\frac{4}{1+4 h^{2}}+\frac{3}{\left(1+4 h^{2}\right)^{2}}\right\}$ and the skew scattering term $\sigma_{x y}^{S K}(H, T \rightarrow 0)=-\eta \frac{e^{2}}{2 \pi \hbar} \frac{h}{\left(1+4 h^{2}\right)^{2}}$. It is interesting to observe that the side-jump term does not depend on the disorder strength. The dimensionless parameter $\eta \equiv \frac{\left[V_{I}^{(1)}\right]^{3} v_{f} k_{f}}{2 \pi n_{I}\left[V_{I}^{(0)}\right]^{4}}$ in the skew scattering term measures the disorder strength in the third order, where $V_{I}^{(1)}$ is a disorder strength of the third order.

Based on Eqs. (3) and (4), we obtain the longitudinal and Hall resistances as follows

$$
\begin{aligned}
& \rho_{x x}(H, T \rightarrow 0)=\frac{\sigma_{x x}(H, T \rightarrow 0)}{\left[\sigma_{x x}(H, T \rightarrow 0)\right]^{2}+\left[\sigma_{x y}(H, T \rightarrow 0)\right]^{2}}, \\
& \rho_{x y}(H, T \rightarrow 0)=\frac{\sigma_{x y}(H, T \rightarrow 0)}{\left[\sigma_{x x}(H, T \rightarrow 0)\right]^{2}+\left[\sigma_{x y}(H, T \rightarrow 0)\right]^{2}} .
\end{aligned}
$$

It should be noted that $\sigma_{x y}$ in the denominator cannot be ignored in this case because this term is comparable to $\sigma_{x x}$. Here we have two dimensionless parameters, $\alpha$ and $\eta$. However, the contribution from the skew scattering turns out to be negligible (SI III). Only one fitting parameter $\alpha$ remains for both MR and Hall resistance.

When either holes (sample $\sharp 1$ and $\sharp 2$ ) or electrons (sample $\sharp 5$ ) are heavily doped, MR curves greatly deviate from the single Dirac-fermion theory (SI III). On the other hand, nearly insulating samples (sample $\sharp 3$ and $\sharp 4$ ) display reasonable matches between experiment and theory. see Fig 4. The Hall resistance also shows deviation from the single Dirac-fermion theory for heavily doped samples (SI III) but not much for nearly insulating samples (sample $\sharp 3$ and $\sharp 4$ ), implying that insulating samples are explained by the theory in a quantitative and consistent way. These results provide a compelling evidence for surface Dirac electrons. In particular, the dominant contribution in the Hall resistance turns out to be the side-jump mechanism at fields below $1 \mathrm{~T}$ and the Berry-curvature effect at higher fields. See Fig. 5 in SI III. 
It is also worth noting that the curvature of the Hall resistance in sample $\sharp 4$ is larger than that in sample $\sharp 3$. According to Dirac theory with disorder, two parameters affect the shape of the Hall resistance: the disorder strength $\alpha$ and the parameter $\gamma$ that measures the distance from the Dirac point. In Fig. 6 of SI III, we show how these parameters influence the curvature of Hall resistance. By decreasing $\gamma$, the Hall resistance becomes straighter because the anomalous Hall effect weakens.

In this letter we have measured the fundamental length scale of the topological insulator, the thickness of the surface state, from the Sondheimer oscillation in magnetoresistance. This surface state is described by the single Dirac-fermion theory. The topological nature of this is verified by the fact that the Hall resistance mainly results from the anomalous Hall effect of Dirac theory, which in turn is dominated by both the side-jump mechanism and the Berry-curvature effect.

The surface thickness will diverge at the critical point of a phase transition from a band insulator to a topological insulator. Such a phase transition was demonstrated in the $\mathrm{HgTe}$ quantum well structure when the size of the quantum well was tuned [25, 26]. On the other hand, the topological phase transition has not yet been achieved in three-dimensional topological insulators. Our measurement for the surface thickness can be utilized as an important tool, revealing the mechanism of such a topological phase transition.

\section{ACKNOWLEDGEMENTS}

This research is supported by Basic Science Research Program through the National Research Foundation of Korea (NRF) funded by the Ministry of Education, Science, and Technology (No. 2010-0021438). K.-S. Kim is supported by the National Research Foundation of Korea (NRF) grant funded by the Korea government (MEST) (No. 2010-0074542).

*hjkim76@daegu.ac.kr; †'sasaki@sci.kj.yamagata-u.ac.jp

[1] V. L. Ginzburg, Rev. Mod. Phys. 76, 981 (2004).

[2] Y. Nambu, Rev. Mod. Phys. 81, 1015 (2009). 
[3] N. Nagaosa, J. Sinova, S. Onoda, S., A. H. MacDonald, and N. P. Ong, Rev. Mod. Phys. 82, 1539 (2010).

[4] D. Xiao, M.-C. Chang, Q. Niu, Rev. Mod. Phys. 82, 1959 (2010).

[5] M. Z. Hasan and C. L. Kane, Rev. Mod. Phys. 82, 3045 (2010).

[6] C. Nayak, S. H. Simon, A. Stern, M. Freedman, and S. D Sarma, Rev. Mod. Phys. 80, 1083 (2008).

[7] R. Shindou, R. Nakai, and S. Murakam, New J. of Phys. 12, 065008 (2010).

[8] L. Fu, C. L. Kane, and E. J. Mele, Phys. Rev. Lett. 98, 106803 (2007).

[9] J. E. Moore and L. Balents, Phys. Rev. B 75, 121306 (2007).

[10] R. Roy, Phys. Rev. B 79, 195322 (2009).

[11] R. Roy, Phys. Rev. B 79, 195321 (2009).

[12] D. Hsieh, et al., Nature 452, 970 (2008).

[13] D. Hsieh et al., Science 323, 919 (2009).

[14] Y. L. Chen et al., Science 10, 178 (2009).

[15] A. H. Castro Neto et al., Rev. Mod. Phys. 81, 109 (2009).

[16] D. Hsieh et al., Nature 460, 1101 (2009).

[17] K. Nomura, M. Koshino, and S. Ryu, Phys. Rev. Lett. 99, 146806 (2007).

[18] E. H. Sondheimer, Phys. Rev. 80, 401 (1950).

[19] A. A. Taskin and Y. Ando, Phys. Rev. B 80, 085303 (2009).

[20] D. Qu et al., Science 329, 821 (2010).

[21] J. G. Analytis et al., Nature Physics 6, 960 (2010).

[22] Z. Ren, et al., Phys. Rev. B 82, 241306 (2010).

[23] C.-L. Song, Preprint at http://arxiv.org/abs/1007.0809 (2010).

[24] N. A. Sinitsyn et al., Phys. Rev. B 75, 045315 (2007).

[25] B. A. Bernevig, T. L. Hughes, and S. C. Zhang, Science 15, 1757 (2006).

[26] M. Konig et al., Science 2, 766 (2007).

[27] Y. S. Hor et al., Phys. Rev. B 79, 195208 (2009). 
FIG. 1: A schematic picture of the surface layer with a length scale $a$ and the Dirac cone at the surface state. a. The schematic diagram for $\mathrm{Bi}_{2} \mathrm{Te}_{3}$ shows the surface layer with thickness $a$. The surface thickness $a$ is determined from Sondheimer oscillation in magnetoresistance, originating from the quantization of motion within the surface layer. b. The Dirac dispersion of the surface state gives rise to topologically nontrivial physical properties. In particular, the anomalous Hall effect due to the Dirac cone turns out to dominate in the Hall resistance. See Fig. 5 in the supplementary information.

FIG. 2: Sondheimer oscillation in magnetoresistance. a. The second derivative of the resistance with respect to the applied magnetic field shows oscillation with a periodicity in $H$, compared to the theoretical curve (red thick line) based on Sondheimer's transport theory. The experimental periodicity matches well with the theoretical curve, but it deviates from theoretical values around $H=4 \mathrm{~T}$. b. Peak and dip number vs. peak and dip position (magnetic fields) in (a). Data points are located on a straight line at low magnetic fields, confirming the $H$ linear periodicity instead of the $1 / H$ periodicity. On the other hand, the experimental data will deviate from the straight line in high magnetic fields due to the appearance of Shubnikov-de Haas oscillation. The second derivative of the magnetoresistance measured up to $55 \mathrm{~T}$ is plotted with respect to $H$ (c) and $1 / H(\mathrm{~d})$. This comparison reveals that the Sondheimer oscillation exists in the region of low magnetic fields while the Shubnikov-de Haas oscillation with a periodicity in $1 / H$ appears at high magnetic fields.

FIG. 3: Dependence of Sondheimer oscillation on the Fermi energy and the disorder strength. a. Dependence of Sondheimer oscillation on the Fermi energy. $\gamma=h / H$ measures the distance of the Fermi energy from the Dirac point, where $h=\hbar \omega_{L} / E_{F}$ is the dimensionless magnetic field given by the ratio of the Zeeman energy $\hbar \omega_{L}$ to the Fermi energy $E_{F}$. Increasing $\gamma$, i.e., as the Fermi surface becomes close to the Dirac point, the period of the Sondheimer oscillation decreases. b. Dependence of Sondheimer oscillation on the disorder strength. $\kappa=a / l$ measures the mean free path. It does not affect the periodicity, changing the amplitude of the oscillation only. c. Peak and dip number vs. peak and dip position (magnetic fields) as a function of $\gamma$ with a fixed $\kappa$. This confirms our conclusion in Fig. 3a. 
FIG. 4: Magnetoresistance and Hall resistance for sample $\sharp 3$ and $\sharp 4$ with theoretical

fitting. The magnetoresistance and Hall resistance of sample $\sharp 3$ are displayed in a and b, respectively, together with theoretical curves (red thick line) based on the single Dirac-fermion theory. The same quantities of sample $\sharp 4$ are presented in $\mathrm{c}$ and $\mathrm{d}$. We emphasize that the theoretical curves for Hall data are based on the parameters from our fitting of magnetoresistance data. In other words, $\gamma$ (the ratio of the Zeeman energy to the Fermi energy) and $\alpha$ (the disorder strength in the longitudinal resistance) are determined from both the Sondheimer oscillation and magnetoresistance completely, and there are no free parameters for the Hall resistance. See the text. Reasonable matches between the experimental data, particulary for the Hall resistance, and the theory reveal that the nature of the surface state is described by the single Dirac-fermion theory. Furthermore, we found that the Berry-curvature term dominates the experimental data of Hall resistance around $3 \mathrm{~T} \sim 4 \mathrm{~T}$ while the side-jump mechanism works around $H=1 \mathrm{~T}$, confirming the topological origin of the transport phenomena in the surface state of topological insulators. See Fig. 5 in the supplementary information. 


\title{
Supplementary information for Sondheimer oscillation as a fingerprint of surface Dirac fermions
}

\author{
Heon-Jung Kim ${ }^{1, *}$, Ki-Seok Kim${ }^{2,3}$, Mun Dae Kim${ }^{4}$, S.-J. Lee ${ }^{5}$, J.-W. Han ${ }^{1}$, \\ A. Ohnishi ${ }^{5}$, M. Kitaura ${ }^{5}$, and M. Sasaki ${ }^{5, \dagger}$, A. Kondo ${ }^{6}$, and K. Kindo ${ }^{6}$ \\ ${ }^{1}$ Department of Physics, College of Natural Science, \\ Daegu University, Gyeongbuk 712-714 Republic of Korea \\ ${ }^{2}$ Asia Pacific Center for Theoretical Physics, \\ POSTECH, Pohang, Gyeongbuk 790-784, Korea \\ ${ }^{3}$ Department of Physics, POSTECH, \\ Pohang, Gyeongbuk 790-784, Korea \\ ${ }^{4}$ Institute of Physics and Applied Physics, \\ Yonsei University, Seoul 120-749, Korea \\ ${ }^{5}$ Department of Physics, Faculty of Science, Yamagata University, \\ Kojirakawa 1-4-12 Yamagata, 990-8560, Japan \\ ${ }^{6}$ Institute for Solid State Physics, University of Tokyo, \\ Kashiwanoha 5-1-5, Kashiwa, Chiba 277-8581 Japan
}

(Dated: October 27, 2018)

\begin{abstract}
In this supplementary information we discuss how to fit our experimental data to theoretical predictions of transport on the surface of $\mathrm{Bi}_{2} \mathrm{Te}_{3}$. First, we describe the sample synthesis and its resulting character. Second, we present the detailed procedure for fitting Sondheimer oscillation data to theory, where the fundamental length scale of the surface state is determined. Third, we discuss the non-oscillatory part of magnetoresistance and Hall resistance based on the single Diracfermion theory, where the topological nature of the surface state is verified. Last, we consider the quantum Hall effect by examining electromagnetic vector potential. The theoretical result from the quantum Hall effect shows that our experimental regime is far from the quantum Hall effect and thus, the effect of the electromagnetic vector potential is negligible in our analysis.
\end{abstract}

PACS numbers: 


\section{SAMPLE PREPARATION AND CHARACTERIZATION}

Single crystals of $\mathrm{Bi}_{2} \mathrm{Te}_{3}$ were grown by a modified bridgeman method, where $\mathrm{Bi}_{2} \mathrm{Te}_{3}$ powder is melted and crystalized in an evacuated quartz ampoule several times by slow cooling. The sample was cooled from $850^{\circ} \mathrm{C}$ to $550^{\circ} \mathrm{C}$ with a cooling rate of $-10 \mathrm{~K} / \mathrm{h}$. Since as-grown $\mathrm{Bi}_{2} \mathrm{Te}_{3}$ is usually p-doped $\left(\mathrm{p}-\mathrm{Bi}_{2} \mathrm{Te}_{3}\right)$ due to the anti-site defects at the $\mathrm{Bi}$ sites [1] , we have controlled the amounts of these defects by adding extra Bi or Te; the doped Bi tends to increase such defects, while the doped Te tends to reduce them. To grow topologically insulating samples $\left(\mathrm{TI}_{-} \mathrm{Bi}_{2} \mathrm{Te}_{3}\right)$, we should add small amounts of $\mathrm{Te}$ to the $\mathrm{p}-\mathrm{Bi}_{2} \mathrm{Te}_{3}$ ingots. In our experiments, we found that $7 \sim 8 \%$ extra Te was necessary in order to synthesize TI- $\mathrm{Bi}_{2} \mathrm{Te}_{3}$. Even if such an ingot were carefully grown, only few single crystals from that ingot showed the desired nonmetallic T-dependent resistivity curves, indicating that large ingots usually contain weak concentration- or defect-gradient [2]. Therefore, we had to select and check every crystal from the large ingots to confirm their non-metallic characteristics. In the selection process, we measured the temperature-dependent resistivity down to $77 \mathrm{~K}$ and thermoelectric power at room temperature of about 200 samples. The $n-\mathrm{Bi}_{2} \mathrm{Te}_{3}$ single crystals were obtained by the further doping of Te to produce the $\mathrm{TI}_{-} \mathrm{Bi}_{2} \mathrm{Te}_{3}$ ingots.

For our experiments, we selected two p- $\mathrm{Bi}_{2} \mathrm{Te}_{3}$ samples (fully p-type $\sharp 1$ and lightly p-type $\sharp 2)$, two $T I-\mathrm{Bi}_{2} \mathrm{Te}_{3}$ samples $(\sharp 3$ and $\sharp 4)$, and one $n-\mathrm{Bi}_{2} \mathrm{Te}_{3}$ sample ( $\left.\sharp 5\right)$. The carrier sign was determined from the sign of the thermopower at room temperature and was also checked using Hall resistivity at $4.2 \mathrm{~K}$, both of which gave the same sign. Magnetoresistance (MR) and Hall effect measurements have been carried out by a six-probe method at $4.2 \mathrm{~K}$, using a superconducting magnet up to $4 \mathrm{~T}$ and a $60 \mathrm{~T}$ pulse magnet at ISSP in the university of Tokyo up to $55 \mathrm{~T}$. Here, the direction of the magnetic field applied is perpendicular to the naturally cleaved plane, on which the current is applied. For MR and Hall measurements, we carefully contacted the lead wires to reduce induction noise for high-field pulse-magnet experiments. We have taken the anti-symmetrized and symmetrized parts in Hall and longitudinal resistances, respectively.

Figure 1 shows the temperature dependency of the resistivity for the p-, TI-, and n- $\mathrm{Bi}_{2} \mathrm{Te}_{3}$ single crystals. The resistivity for the both $\mathrm{p}$ - and n-type samples $(\sharp 1, \sharp 2$, and $\sharp 5)$ decreases monotonically with decreasing temperature, which is a typical metallic characteristic, while the resistivity for the TI samples $(\sharp 3$ and $\sharp 4)$ increases below $\sim 180 \mathrm{~K}$ and then tends to 


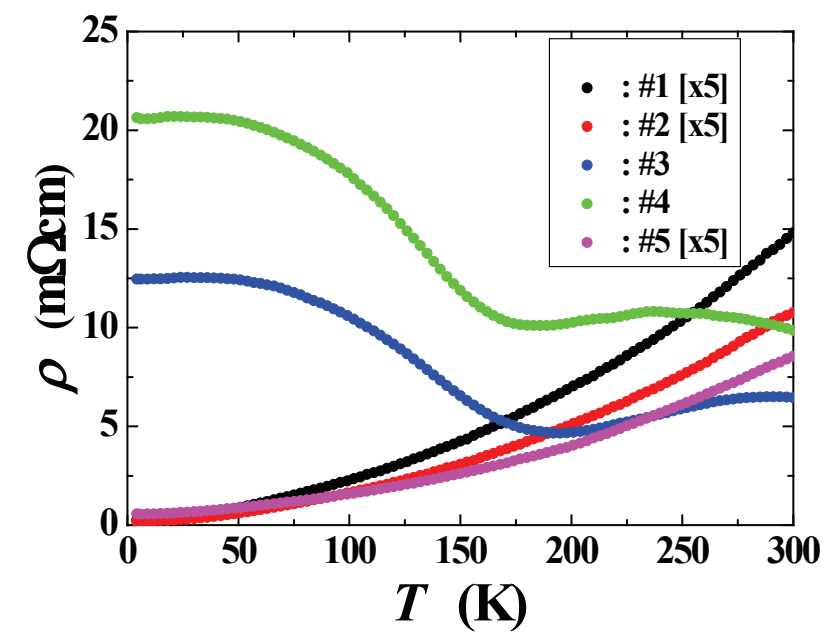

FIG. 1: Temperature dependency of resistivity for samples $\sharp 1-\sharp 5$.

saturate below $50 \mathrm{~K}$. The magnitude of the resistivity for the $\mathrm{TI}^{-\mathrm{Bi}_{2}} \mathrm{Te}_{3}$ single crystals is $5 \sim 10$ times larger than those for the p- and n-type samples. The non-metallic nature observed for the TI samples is consistent with those reported by the Princeton group [2]. The oscillating part of MR was separated by fitting the background with a polynomial.

We have analyzed all five samples and discussed them in both the main text and the supplementary information. Among them, the TI samples ( $\sharp 3$ and $\sharp 4)$ are best fitted to the single Dirac-fermion theory while the fitting becomes worse for the p- and $n-\mathrm{Bi}_{2} \mathrm{Te}_{3}$ samples. Deviation between the data of samples $\sharp 1, \sharp 2$, and $\sharp 5$ and the theoretical curves can be seen in Fig. 3 and Fig. 4. These figures clearly show that the linear part in MR of the experimental data deviates from the theoretical fitting significantly, implying that bulk channels for conduction are important beyond the single Dirac-fermion theory. Judging from our analysis, the Fermi level of the surface states for sample $\sharp 3$ and $\sharp 4$ lies below the Dirac point, corresponding to hole doping, while that in the bulk is between the bulk gap. The difference of the Fermi level between the surface and bulk originates from a band bending as reported before [3, 4]. On the other hand, the bulk Fermi level is below the valence band maximum for sample $\sharp 1$ and $\sharp 2$. This explains the metallic properties and the disagreement between theory and experimental data for samples $\sharp 1, \sharp 2$, and $\sharp 5$. 


\section{SONDHEIMER OSCILLATION}

Sondheimer oscillation appears in metallic thin films, where a finite thickness of a surface state gives rise to a quantization of motion, resulting in an oscillation in MR and Hall resistance [5]. While Shubnikov-de Haas oscillation displays periodicity proportional to $1 / H$, the periodicty of Sondheimer oscillation is proportional to $H$. This periodicity allows us to find the thickness of the surface state. Although $\mathrm{Bi}_{2} \mathrm{Te}_{3}$ is a bulk material (semiconductor), the topological structure of the ground state gives rise to a surface state with thickness $a$, which is protected from time-reversal invariant perturbations [6-9]. In this respect, the Sondheimer oscillation is undisputable evidence for existence of a surface state.

We start from the Boltzmann equation

$$
-\frac{e}{\hbar}\left(\boldsymbol{E}+\frac{\overline{\boldsymbol{v}}}{c} \times \boldsymbol{H}\right) \cdot \nabla_{\boldsymbol{k}} f+\overline{\boldsymbol{v}} \cdot \nabla_{\boldsymbol{r}} f=-\frac{f-f_{0}}{\tau} .
$$

$f=f_{0}+f_{1}(\overline{\boldsymbol{v}}, z)$ is a non-equilibrium distribution function with its equilibrium part $f_{0}$. The non-equilibrium part $f_{1}$ depends on the $z$ coordinate. $\overline{\boldsymbol{v}}=\hbar \overline{\boldsymbol{k}} / m^{*}$ is the average velocity, where $m^{*}$ is an effective mass of the surface Dirac electrons, and $\overline{\boldsymbol{k}}$ is the average momentum, which is determined later. The dispersion is given by $\epsilon_{\boldsymbol{k}}=-\mu+\sqrt{v_{f}^{2} k^{2}+\left(g^{*} H\right)^{2}}$ in the presence of a z-directional magnetic field $H$, where $v_{f}$ is the Dirac velocity and the Fermi momentum is $k_{f}=\frac{\sqrt{\mu^{2}-\left(g^{*} H\right)^{2}}}{v_{f}} . \mu$ is the chemical potential at the surface, and $g^{*}$ is the Lande-g factor of the surface electron. $\boldsymbol{H}=H \hat{z}$ is an applied magnetic field in the $z$ direction and $\boldsymbol{E}=E_{x} \hat{x}+E_{y} \hat{y}$ is an electric field, where a $y$-directional electric field is induced. $\tau$ is the mean-free time, which measures the strength of disorder.

Following the same procedure as in the original paper of Sondheimer [5], we obtain

$$
\rho(H, T)=\frac{\rho_{0}}{\kappa} \Re \phi(s),
$$

where $\rho(H, T)$ is the resistivity. $\rho_{0}=\frac{m^{*}}{n e^{2} \tau}$ is the residual resistivity with the density $n=$ $\frac{8 \pi}{3}\left(\frac{m^{*} \bar{v}}{2 \pi \hbar}\right)^{3}$ of surface electrons, and $\kappa=\frac{a}{l}$, where $l=\bar{v} \tau$ is the mean free path and $a$ is the surface thickness, determined from fitting. $\phi(s)$ results from the distribution function in the hard-wall boundary condition for the $z$ direction, given by

$$
\frac{1}{\phi(s)}=\frac{1}{s}-\frac{3}{8 s^{2}}+\frac{3}{2 s^{2}} \int_{1}^{\infty} d u e^{-s u}\left(\frac{1}{u^{3}}-\frac{1}{u^{5}}\right)
$$

after integrating over z. $s=\kappa+i \beta$, where $\beta=\frac{a}{r_{c}}$ with the magnetic length $r_{c}=\frac{m^{*} \bar{v} c}{e H}$. 
For the numerical analysis, it is important to express the $\beta$ variable in terms of dimensionless parameters because the surface thickness $a$ is determined from an appropriate choice of one parameter in $\beta$, referred to as $\gamma$, which will be discussed later. It is given by

$$
\beta=\frac{1}{2}\left(k_{f} a\right) \frac{\hbar \omega_{L}}{E_{F}} \frac{k_{f}}{\bar{k}}
$$

where $\hbar \omega_{L}=\hbar \frac{e g^{*} H}{2 m^{*} c}$ is the effective Zeeman energy and $E_{F}=\frac{k_{f}^{2}}{2 m^{*}}$ is the Fermi energy with an effective mass $m^{*} . \bar{k} / k_{f}$ is determined from

$$
\frac{\bar{k}}{k_{f}}=\frac{1}{t} \int_{0}^{\infty} d \epsilon \frac{\epsilon}{\sqrt{\epsilon^{2}+h^{2}}} \frac{e^{\frac{\sqrt{\epsilon^{2}+h^{2}}-\mu^{\prime}}{t}}}{\left(e^{\frac{\sqrt{\epsilon^{2}+h^{2}}-\mu^{\prime}}{t}}+1\right)^{2}},
$$

where we introduce several dimensionless parameters, scaled by the Fermi energy, such that an effective dispersion $\epsilon=v k / E_{F}$ with $v=\frac{\hbar^{2} k_{f}}{2 m^{*}}$, the Zeeman energy $h=\hbar \omega_{L} / E_{F}$, the chemical potential $\mu^{\prime}=\mu / E_{F}=\sqrt{1+h^{2}}$, and an effective temperature $t=T / E_{F}$.

Based on this formulation, we fit the oscillation data of MR. See Fig. 2 for sample $\sharp 2$. First, we determine $\kappa \approx 0.02$, which characterizes the strength of the disorder for the best match of the amplitude with the Sondheimer oscillation. It is of note the matching cannot be perfect. In fact, the variation of $\kappa$ does not modify the periodicity of the Sondheimer oscillation although it changes the oscillation amplitude.

The surface thickness is determined from the periodicity $\Delta H$ in the experimental data. Considering that Eq. (5) fixes the ratio of $\bar{k} / k_{f}$, two parameters in $\beta$ remain unknown, which correspond to $k_{f} a$ and $\gamma$ which is the ratio $\hbar \omega / E_{F}$ at $H=1 \mathrm{~T}$. Therefore, we cannot determine the thickness of the surface state from the oscillation period alone. As discussed in the main text, the same value of $\gamma$ is utilized for both the non-oscillatory part of MR and Hall resistance. If the value of $\gamma$ were incorrectly chosen, we could not fit both the nonoscillatory part of MR and Hall resistance consistently. See Fig. 6-b. Combined with both longitudinal and transverse resistances, we found that $\gamma \approx 0.44$ fits the experimental data well. This value is quite interesting because it almost coincides with the $\gamma$ value determined from the values of the bulk parameters. For example, if we use bulk values of the Lande- $g$ factor and effective mass, which are $g^{*} \approx 13.7$ and $m^{*} \approx 0.1 m_{e}\left(m_{e}\right.$ is the bare mass of an electron), we obtain $\hbar \omega_{L} \approx 100 \mathrm{~K}$ at $H=1 \mathrm{~T}$, giving rise to $h \approx 0.44$ with a typical bulk value for the Fermi energy $E_{F} \approx 230 \mathrm{~K}$. Since we cannot determine $g^{*}, m^{*}$, and $E_{F}$ at the surface from our experiment, each value at the surface may not be the same as the bulk one. 

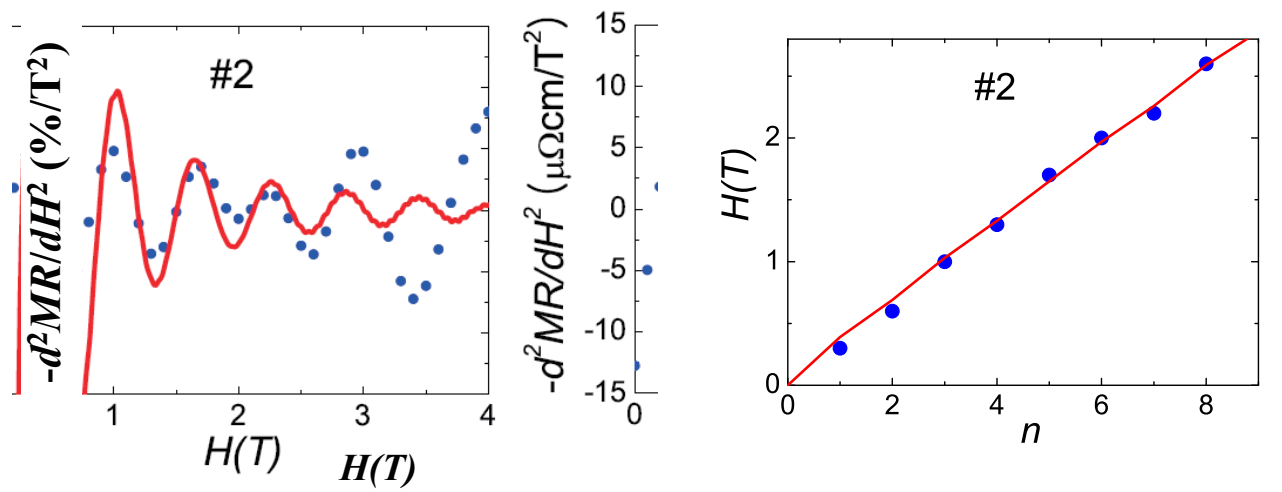

FIG. 2: a. Sondheimer oscillation for sample $\sharp 2$. The periodicity is well matched, but the oscillation amplitude deviates rather a lot due to the appearance of the Shubnikov-de Haas oscillation above 4 T. Sondheimer oscillations are also observed for the p- and n-doped samples, where the surface channel for conduction coexists with the bulk conduction. b. Peak and dip number vs. peak and dip position (magnetic fields) in (a). Data points are located on a straight line, confirming the $H$ linear periodicity instead of the $1 / H$ periodicity.

However, it should be noted that our $\gamma$ seems to be universal in both $\mathrm{Bi}_{2} \mathrm{Te}_{3}$ and $\mathrm{Bi}_{2} \mathrm{Se}_{3}$ although both $g^{*}$ and $E_{F}$ in $\mathrm{Bi}_{2} \mathrm{Se}_{3}$ are almost four times larger than those in $\mathrm{Bi}_{2} \mathrm{Te}_{3}$ [3].

\section{MAGNETORESISTANCE AND HALL EFFECT}

Theoretically, the longitudinal resistance results from the transport of electrons near the Fermi surface, and is described quasi-classically or quantum-mechanically. In this case, the quasi-classical treatment based on the Boltzmann equation gives the same result as the quantum mechanical treatment based on the Kubo-formula. On the other hand, there are various contributions with a topological origin in the Hall resistance, which is beyond the conventional treatment used in the Boltzmann equation approach. The Boltzmann equation needs additional terms [11] in order to mimic the Kubo-formula [12, 13].

Since several terms exist in the Hall resistance, which are explained in the main text, an important question is which terms give a large contribution to the Hall resistance. We explicitly demonstrate that the side-jump mechanism dominates in low magnetic fields while the Berry-curvature effect dominates the Hall resistance at high magnetic fields (Fig. 5). In contrast, the skew scattering term turns out to be negligible. We also check the dependence 

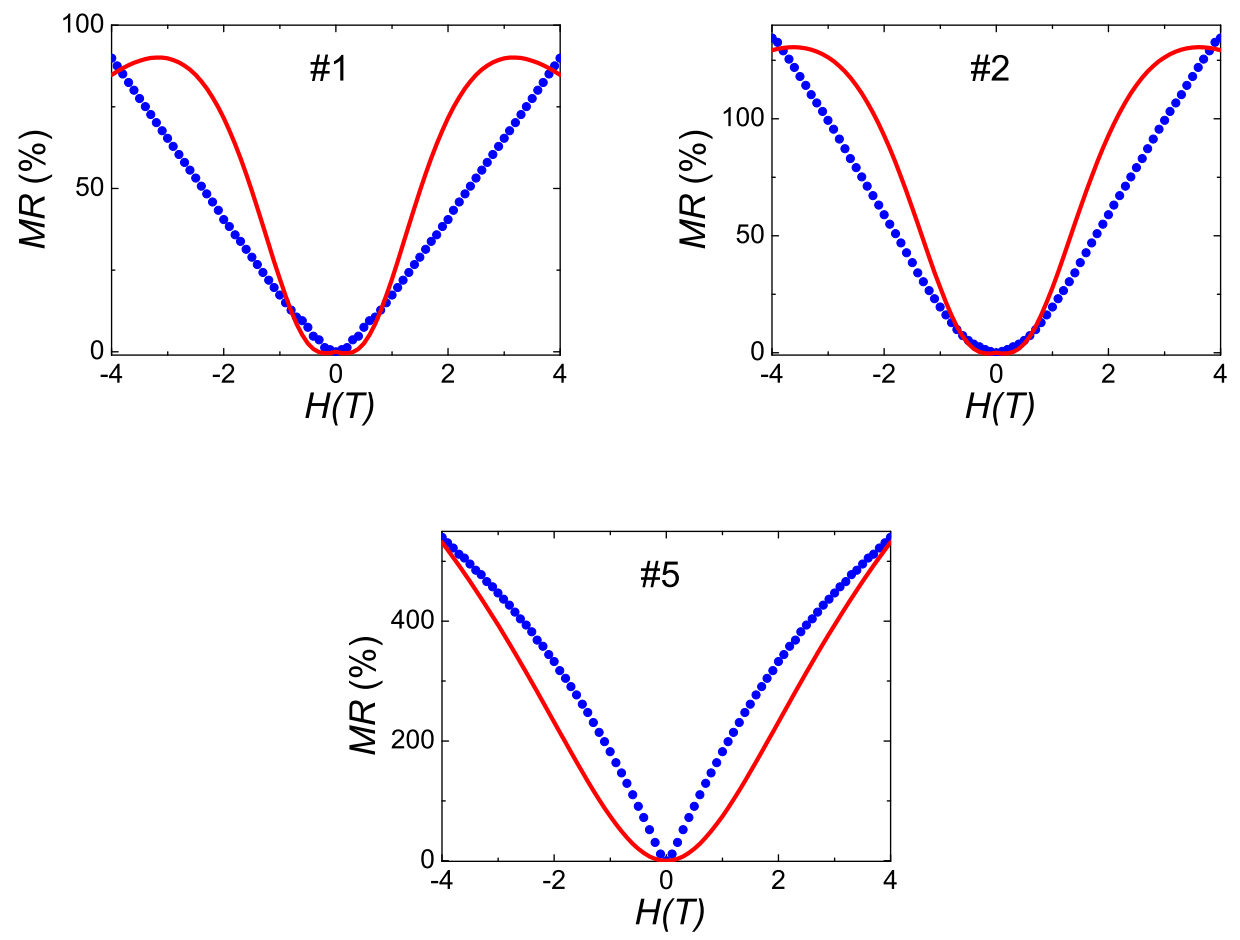

FIG. 3: Magnetoresistance for a. $\sharp 1$, b. $\sharp 2$, and c. $\sharp 5$, where the disorder strength is utilized as a fitting parameter, given by $\alpha \approx 0.4, \alpha \approx 0.5$, and $\alpha \approx 1.5$, respectively. As discussed in section I, the presence of bulk conduction channels does not allow us to describe these samples purely within the single Dirac-fermion theory.

of the Hall resistance on either the disorder strength $\alpha$ (Fig. 6-a) or the parameter $\gamma$, which determines the Fermi level (Fig. 6-b). Roughly speaking, to vary $\gamma$ is to rescale the $x$-axis while to change $\alpha$ is to rescale the $y$-axis. Figure 6 -a and Fig. 6-b show how these parameters affect the shape of the Hall resistance. These results confirm that if we do not choose the correct $\gamma$, it is difficult to get a reasonable match between the experiment and theory.

\section{CONTRIBUTION FROM LANDAU LEVEL}

In the Sondheimer oscillation we have pointed out that the predicted oscillation amplitude does not match with the experimental data because of the Shubnikov-de Haas oscillation which occurs above $4 \mathrm{~T}$. In order to confirm irrelevance of the formation of Landau levels at low magnetic fields, we take into account the vector potential for the MR and Hall resistance. 

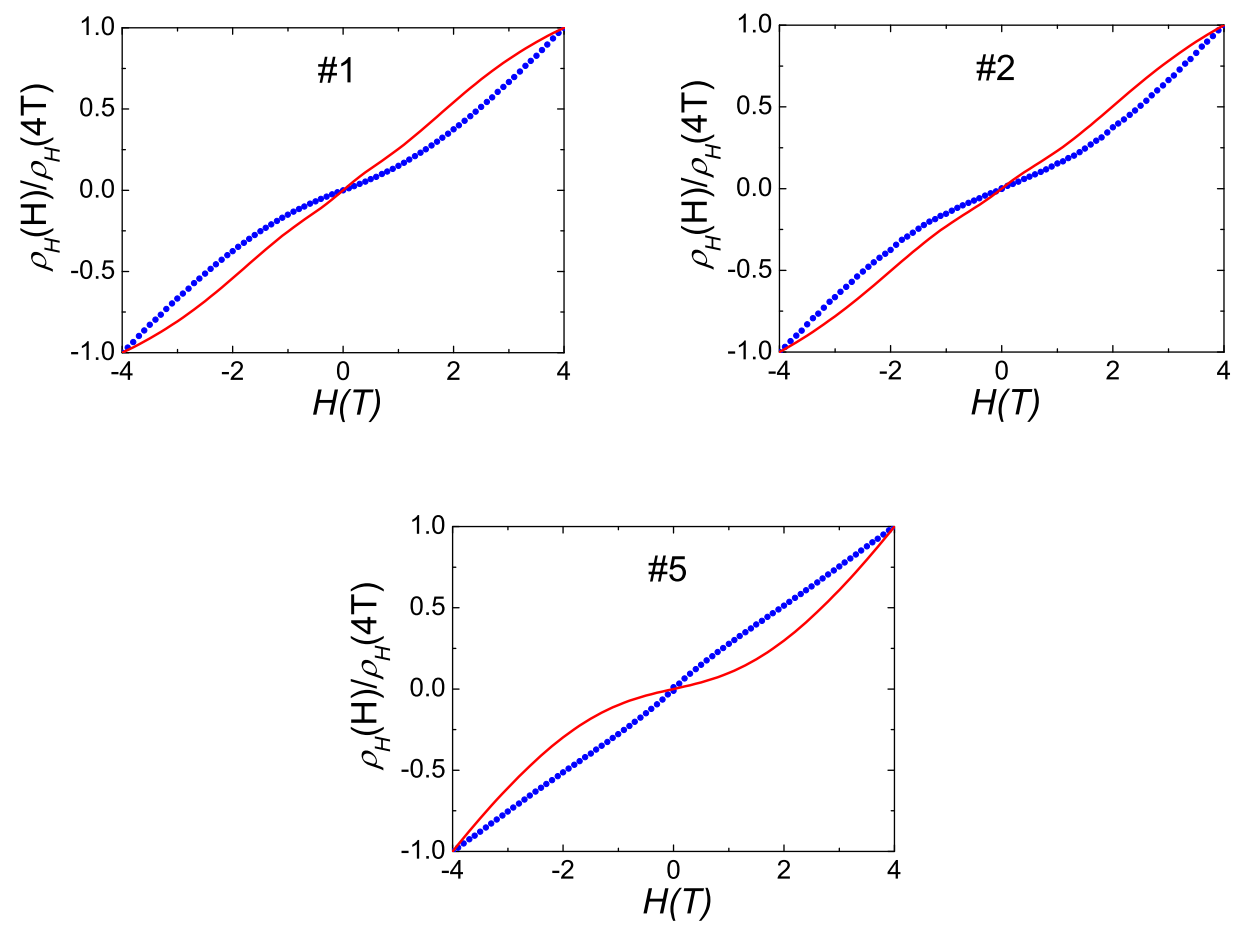

FIG. 4: Hall resistance for a. $\sharp 1, \mathrm{~b} . \sharp 2$, and $\mathrm{c} \sharp 5$, where the same strength of disorder is utilized as in Fig. 3, respectively. We believe that the origin of this mismatch between experiment and theory lies in the contribution from the bulk transport which is not included in the single Dirac-fermion theory.

This has been performed in the context of the quantum Hall effect in graphene, where the two valley contributions are simply added [14].

The longitudinal conductance is given by [14]

$$
\begin{aligned}
\sigma_{x x}(H, T) & =\frac{e^{2} N_{f} \Gamma}{4 \pi^{2} T} \int_{-\infty}^{\infty} d \omega \frac{1}{\cosh ^{2}\left(\frac{\omega+\mu}{2 T}\right)} \frac{\Gamma}{\left(\frac{v_{f}^{2} e H}{c}\right)^{2}+(2 \omega \Gamma)^{2}} \\
& \times\left[2 \omega^{2}+\frac{\left(\omega^{2}+\Delta^{2}+\Gamma^{2}\right)\left(\frac{v_{f}^{2} e H}{c}\right)^{2}-2 \omega^{2}\left(\omega^{2}-\Delta^{2}+\Gamma^{2}\right)\left(\frac{v_{f}^{2} e H}{c}\right)}{\left(\omega^{2}-\Delta^{2}-\Gamma^{2}\right)^{2}+4 \omega^{2} \Gamma^{2}}\right. \\
& \left.-\frac{\omega\left(\omega^{2}-\Delta^{2}+\Gamma^{2}\right)}{\Gamma} \Im \Psi\left(\frac{\Delta^{2}+\Gamma^{2}-\omega^{2}-2 i \omega \Gamma}{2 v_{f}^{2}|e H| / c}\right)\right]
\end{aligned}
$$

with $N_{f}=1$ ( $N_{f}=2$ for graphene), where $\Delta=\hbar \omega_{L}$ is the Zeeman energy, $\Gamma$ is the imaginary part of the electron self-energy due to disorder, and $\Psi(z)$ is the digamma function. 


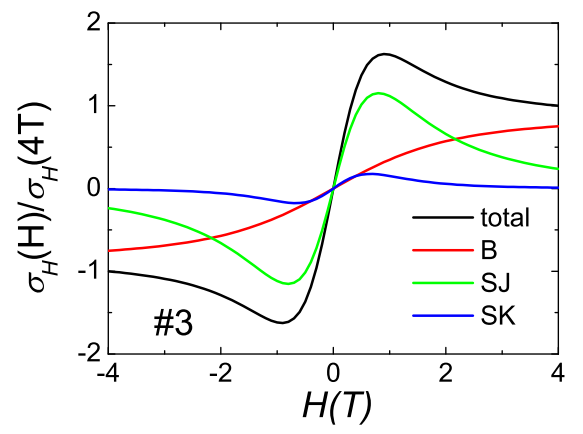

FIG. 5: Each contribution in Hall conductance shows that the dominant term is the side-jump term in low fields and the Berry curvature term in high fields. These curves are generated, by using the parameter values from sample $\sharp 3, \alpha=0.5$ and $\gamma=0.44$.
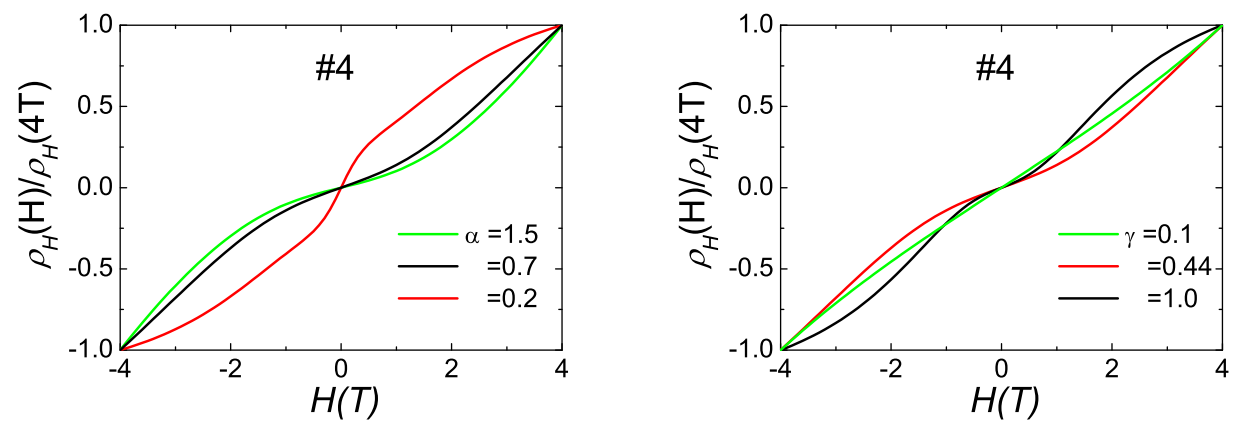

FIG. 6: a. Hall resistance deviates from the $\alpha=0.7$ line with $\gamma=0.44$, changing $\alpha$. b. Hall resistance separates from the $\gamma=0.44$ line with $\alpha=0.7$, varying $\gamma$. The Hall resistance of sample $\sharp 4$ is well fitted by using $\alpha=0.7$ and $\gamma=0.44$.

The Hall conductance is [14]

$$
\sigma_{x y}(H, T)=\frac{e^{2} N_{f}}{2 \pi} \nu_{B}
$$

where $\nu_{B}$ is the filling factor, given by

$$
\begin{aligned}
\nu_{B} & =\int_{-\infty}^{\infty} \frac{d \omega}{2 \pi} \tanh \left(\frac{\omega+\mu}{2 T}\right)\left[\frac{\Gamma}{(\omega-\Delta)^{2}+\Gamma^{2}}+(\omega \longleftrightarrow-\omega)\right. \\
& \left.+2 \sum_{n=1}^{\infty}\left(\frac{\Gamma}{\left(\omega-M_{n}\right)^{2}+\Gamma^{2}}+(\omega \longleftrightarrow-\omega)\right)\right],
\end{aligned}
$$

where $M_{n}=\sqrt{\Delta^{2}+2 n v_{f}^{2}|e H| / c}$ is the dispersion of surface Dirac electrons in the presence of the Landau level. 


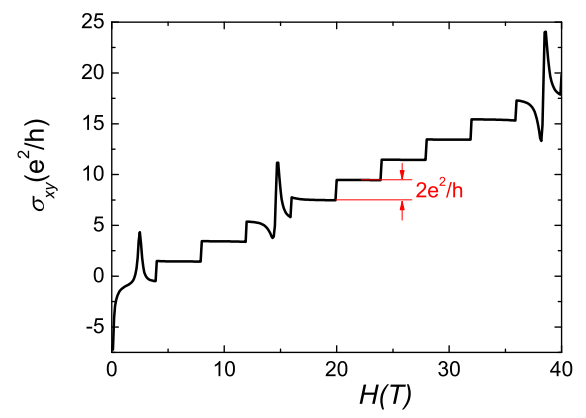

FIG. 7: Quantized Hall conductance, expected to be relevant in high magnetic fields.

By using these expressions, we plot the Hall conductance in Fig. 7. First of all, the plateau in the Hall conductance is clearly shown. This behavior is far from that given by the experimental data. Therefore, we conclude that our regime is far from being described by the quantum Hall effect. The introduction of the Zeeman term is sufficient to explain the transport data at low magnetic fields.

*hjkim76@daegu.ac.kr; ${ }^{\dagger}$ sasaki@sci.kj.yamagata-u.ac.jp

[1] Y. S. Hor et al. P-type $\mathrm{Bi}_{2} \mathrm{Se}_{3}$ for topological insulator and low-temperature thermoelectric applications Phys. Rev. B 79, 195208 (2009).

[2] Dong-Xia Qu, Y. S. Hor, Jun Xiong, R. J. Cava and N. P. Ong Quantum Oscillations and Hall Anomaly of Surface States in the Topological Insulator $\mathrm{Bi}_{2} \mathrm{Te}_{3}$. Science 329, 821 (2010).

[3] Analytis, J. G. et al. Two-dimensional surface state in the quantum limit of a topological insulator. Nature Physics 6, 960 (2010).

[4] Hsieh, D. et al. Observation of Time-Reversal-Protected Single-Dirac-Cone TopologicalInsulator States in $\mathrm{Bi}_{2} \mathrm{Te}_{3}$ and $\mathrm{Sb}_{2} \mathrm{Te}_{3}$. Phys. Rev. Lett. 103, 146401 (2009).

[5] Sondheimer, E. H. The Influence of a Transverse Magnetic Field on the Conductivity of Thin Metallic Films. Phys. Rev. 80, 401 (1950).

[6] Fu, L., Kane, C. L., \& Mele, E. J. Topological Insulators in Three Dimensions. Phys. Rev. Lett. 98, 106803 (2007).

[7] Moore, J. E. \& Balents, L. Topological invariants of time-reversal-invariant band structures. 
Phys. Rev. B 75, 121306 (2007).

[8] Roy, R. Topological phases and the quantum spin Hall effect in three dimensions. Phys. Rev. $B$ 79, 195322 (2009).

[9] Roy, R. $\mathrm{Z}_{2}$ classification of quantum spin Hall systems: An approach using time-reversal invariance. Phys. Rev. B 79, 195321 (2009).

[10] Song, C.-L. et al. Topological insulator $\mathrm{Bi}_{2} \mathrm{Se}_{3}$ thin films grown on double-layer graphene by molecular beam epitaxy. Preprint at http://arxiv.org/abs/1007.0809 (2010).

[11] Sinitsyn, N. A. et al. Anomalous Hall effect in a two-dimensional Dirac band: The link between the Kubo-Streda formula and the semiclassical Boltzmann equation approach. Phys. Rev. B 75, 045315 (2007).

[12] Nagaosa, N., Sinova, J., Onoda, S., MacDonald, A. H. \& Ong, N. P. Anomalous Hall effect. Rev. Mod. Phys. 82, 1539 (2010).

[13] Xiao, D., Chang, M.-C. \& Niu, Q. Berry phase effects on electronic properties. Rev. Mod. Phys. 82, 1959 (2010).

[14] Gorbar, E. V., Gusynin, V. P., Miransky, V. A. \& Shovkovy, I. A. Magnetic field driven metal-insulator phase transition in planar systems. Phys. Rev. B 66, 045108 (2002). 